Rapid Reviews COVID-19•

\title{
Review 1: "VALIDATION OF A SALIVA-BASED TEST FOR THE MOLECULAR \\ DIAGNOSIS OF SARS-COV-2 INFECTION"
}

\section{Jianhui Wang}

Published on: Oct 21, 2021

License: Creative Commons Attribution 4.0 International License(CC-BY 4.0). 


\section{$\underline{\text { RR:C19 Evidence Scale rating by reviewer: }}$}

- Reliable. The main study claims are generally justified by its methods and data. The results and conclusions are likely to be similar to the hypothetical ideal study. There are some minor caveats or limitations, but they would/do not change the major claims of the study. The study provides sufficient strength of evidence on its own that its main claims should be considered actionable, with some room for future revision.

$* * * * * * * * * * * * * * * * * * * * * * * * * * * * * * * * * * * * * * *$

Review: The manuscript shows a useful saliva-based validation study with comparison data from both saliva and the golden standard samples - NP swab samples. It shows the potential use of the saliva samples in population screening since the saliva samples are more accessible. They have very good sensitivity and specificity, and decent reproducibility due to the feature of their stability. In my opinion, it can be considered as publishable if the authors solve some of my questions as below:

1. The authors collected samples from previously tested patients but did not mention the previous testing methods for the patients. Are those diagnostic tests and screening tests certain PCR-based tests on NP swabs? The authors' testing methods in this study, including RNA extraction and PCR test on NP swabs, should be both validated and confirmed to have the similar accuracy to the previous tools, or the authors should quote a reference as a bridge study to show their test's accuracy. Otherwise, the authors should at least calculate their current test's sensitivity and specificity, by using the data of the cases that they used in the study to compare to the previous testing results.

2. Since there are Ct value related viral load evaluations in the study, it is necessary to briefly mention the volume of saliva collected and the input volume for the RNA extraction. This is also helpful for understanding the settings of the LoD in the assay and the association between viral load and severity, and comparing these datasets to the ones in other studies.

3. What are the sampling criteria for the diagnosed and symptomatic cases? Is there a sampling window according to the onset of symptom, hospital admission or ICU? It is important to describe the sampling time since the viral loads change along with the course of disease. 
4. It is interesting that the saliva in stabilizing saline has higher viral load than the fresh saliva does. Since the authors used $24 \mathrm{~h}$ and $48 \mathrm{~h}$ time points in the sample storage study, the data of Ct values should be displayed. Is there any growing trend along with time? This data may reveal if the virus possibly replicates in the cells in the stabilizing saline, in the sense of when the conserving saline does not deactivate the virus or lyse the cells. 